\title{
Die Lust am Wettkampf
}

\section{Niko Kappel}

Niko Kappel ist ein paralympischer Leichtathlet der Startklasse F41 (Kleinwüchsige Atbleten). Im Kugelstoßen gewann er bei den Paralympics 2016 in Rio de Janeiro die Goldmedaille sowie 2017 die Weltmeisterschaft in London. Zwei Mal wurde er zum Deutschen Behindertensportler des Jahres gekürt.

Wenn ich meinen Werdegang betrachte, hatte Sport schon immer einen sehr hohen Stellenwert in meinem Leben, fast im Gegensatz zur Tatsache, dass ich kleinwüchsig bin. Ich habe schon sehr früh gemeinsam mit allen anderen Fußball im Verein gespielt oder Leichtathletik betrieben. Inklusive Sportangebote gab es damals in der heutigen Form kaum und hätte ich auch nie für nötig befunden. Im Schwimmbad gab es vielleicht schon hin und wieder den ein oder anderen verwunderten Blick, wenn wir als Gruppe kamen - aber ich habe die Blicke damals, auch wenn das komisch klingt, nie auf mich bezogen. Auf die Idee, dass ein Kleinwüchsiger aus dem Rahmen dessen fällt, was die Leute zu sehen gewohnt sind, bin ich nicht gekommen.

Auch von sportlichen Hürden im wahrsten Sinne des Wortes habe ich mich nie abhalten lassen: Bockspringen war sicherlich nicht meine Paradedisziplin, aber es gab ja genug andere Herausforderungen wie etwa Bodenturnen, bei denen ich glänzen konnte. Neben meinem Elternhaus hat Sport sicherlich einen großen Anteil daran, dass ich meinen Weg immer mit einem gesunden Selbstbewusstsein gehen konnte. Ich habe früh gelernt: Jeder kann irgendetwas nicht und irgendetwas besonders gut. Sport ist diesbezüglich zwar erst einmal knallhart, aber er gibt einem eben auch den Anstoß, sich mit eigenen Stärken und Schwächen auseinanderzusetzen. Würden Marcel Nguyen und Robert Harting die Sportart tauschen, hätte das sicherlich einen gewissen Unterhaltungswert - Weltspitze erreichen aber auch diese beiden Ausnahmeathleten eben nur in ihrer jeweiligen Paradedisziplin. Mit der Tatsache, dass ich wohl keine Karriere im Basketball hinlegen werde, hatte ich jedenfalls früh meinen Frieden gemacht.

Mich auf einer gemeinsamen Bühne mit anderen Athleten zu messen, die über ähnliche Ausgangsbedingungen verfügen wie ich, war meine große Motivation zum Einstieg in den paralympischen Sport. Das große 
Ganze hatte ich als junger Athlet nicht im Blick, das kam erst nach und nach. Mir ging es zunächst vor allem um den Wettkampf. Im Laufe der Jahre habe ich aber mehr und mehr realisiert, wie sehr gerade Themen wie Fair Play oder die Olympischen Werte in die Gesellschaft abstrahlen können und welche Rolle dabei Olympische Spiele und Paralympics als Aushängeschilder spielen. Als ich nach der Rückkehr aus Rio de Janeiro 2016 auf einmal zum Bundespräsidenten eingeladen wurde, ist mir diese Dimension erst so richtig bewusst geworden.

Möchte man die Olympischen Werte wirklich dauerhaft im Handeln von Kindern und Jugendlichen verankern, ist es in meinen Augen der falsche Weg, sie ausschließlich theoretisch zu thematisieren. Zentral ist es doch, dass sie im Sport erlebt werden. Der Schulsport spielt dabei für mich nach wie vor eine entscheidende Rolle. Auch wenn dessen Standing gerade in der aktuellen Zeit nicht besonders hoch ist: Im Schulsport werden für mich unglaublich viele soziale Kompetenzen entwickelt, die mindestens so wichtig sind wie mathematische Formeln. Der Respekt und gegenseitige Rücksichtnahme, Fair Play und der Umgang mit Niederlagen werden hier spielerisch eingeübt. Vor allem letzteres finde ich sehr entscheidend: Wenn ich nicht im Sport lerne, zu verlieren - wo soll ich es denn sonst lernen? Wenn ich jedenfalls für mich persönlich reflektiere, welche Aspekte mich in meiner Schulzeit am meisten geprägt haben und was bis heute geblieben ist, dann nehmen Werte und Erfahrungen aus dem Sport einen deutlich größeren Raum ein als das Wissen aus Lehrbüchern.

Natürlich haben auch wir Athlet*innen eine wichtige Rolle, wenn es um die Vermittlung der Werte geht. Wir stehen als Vorbilder im Rampenlicht und tragen damit eine besondere Verantwortung für unser Verhalten. Viele Menschen sehen zu uns auf und orientieren sich an dem, was wir tun. Das heißt aber nicht, dass wir keine Fehler machen dürfen. Fehler sind menschlich und kommen vor. Viele von uns leben Emotionen und stehen oft unter hohem Leistungsdruck. Es ist fast nicht zu verhindern, dass man angesichts dieser Bedingungen irgendwann einmal ein bisschen aus der Rolle fällt. Wichtig ist dann aber die Reaktion: Wie gehe ich damit um, wie positioniere ich mich auch öffentlich? So kann man vielleicht mit einem Fehler beziehungsweise dem reflektierten Umgang damit dann auch noch Positives bewirken.

Die Goldmedaille bei den Paralympics in Rio 2016 hat meine sportlichen Ambitionen nicht geschmälert, im Gegenteil. Ich stecke mitten in der Vorbereitung auf die Wettkämpfe in Tokio und habe mich auch durch den Lockdown nicht aus dem Tritt bringen lassen. Auf unvorhergesehene Entwicklungen muss man mit einer gewissen Flexibilität reagieren, ohne deswegen gleich alle Pläne zu ändern. Erfolg stellt sich für mich oft vor 
allem dann ein, wenn man seinen Weg mit einer gewissen Konsequenz geht.

Die Paralympics entwickeln sich stetig weiter und die Leistungsdichte steigt konstant an. Die Luft an der Spitze wird angesichts der nachrückenden starken Konkurrenz immer dünner, und ich genieße das. Denn nach wie vor ist der Wettkampf das, wofür ich brenne - und je spannender es wird, desto besser. Meine zweite Goldmedaille habe ich jedenfalls fest im Blick. 
